\title{
Sodium butyrate protects against oxidative stress between obesity-prone and obesity-resistant rats induced by HFD through modulating Nrf2 pathway and mitochondrial function
}

\author{
Yipin Lu \\ Jiangnan university \\ Yongjuan Sun \\ Jiangnan University \\ Yingrui Li \\ Jiangnan University \\ Shuhua Ma \\ Jiangnan University \\ Kai Zhang \\ Jiangnan University \\ xue Tang ( $\boldsymbol{\nabla}$ tangxue@jiangnan.edu.cn ) \\ Jiangnan University https://orcid.org/0000-0002-3280-8392 \\ Renqiang Yu \\ The affiliated Wuxi maternity and child health care hospital of nanjing medical university
}

\section{Research}

Keywords: Sodium butyrate, Oxidative stress, Mitochondrial function, Obesity-prone, Obesity-resistant

Posted Date: July 30th, 2020

DOI: https://doi.org/10.21203/rs.3.rs-44355/v1

License: (c) (i) This work is licensed under a Creative Commons Attribution 4.0 International License. Read Full License 


\section{Abstract \\ Background}

Sodium butyrate $(\mathrm{NaB})$ is obtained by fermenting dietary fiber via intestinal microflora and has recently been shown to improve some antioxidant enzymes in vivo.

\section{Methods}

In this study, we investigated the antioxidant effects of Sodium butyrate on obesity rats induced by highfed diet.

\section{Results}

$\mathrm{NaB}$ intervention could effectively reduce the body weight of rats in the obesity-prone(OP) and obesityresistant(OR)group, and reduce insulin resistance, plasma lipid, protect the gastrocnemius muscle and pancreas from oxidative stress induced by high fat diet, upregulate T-AOC, antioxidant enzyme activity and GSH/GSSG ratio, reduce ROS level and MDA content. NaB may increase Pi3k, Nrf2, Nqo-1, Ho-1 and inhibit Gsk-3 $\beta$ mRNA expression by regulating Nrf2 antioxidant pathway to enhance tissue antioxidant capacity. At the same time, NaB intervention could significantly increase the expression of Glut4 and Irs-1 mRNA in gastrocnemius muscle of OP and OR rats, suppress the expression of Bax and Caspase 3, increase the mRNA expression of $P d x 1, M a f A$ and $B c l-2$, and increase insulin secretion and muscle insulin sensitivity. The regulatory effect of $\mathrm{NaB}$ was correlated with its significantly increased activity of mitochondrial antioxidant enzymes, mitochondrial membrane potential, NADH/NAD ${ }^{+}$ratio, acetyl-CoA and ATP production, Tfam and Pgc-1a expression and mitochondrial DNA copy number. In addition, $6 \%$ $\mathrm{NaB}$ intervention in OP rats, $4 \% \mathrm{NaB}$ intervention in OR rats were more significant, indicating that there were dose effects of $\mathrm{NaB}$ intervention in different obesity phenotypes.

\section{Conclusions}

$\mathrm{NaB}$ activates the Nrf2 antioxidant pathway, enhances the expression level of downstream antioxidant genes, improves the antioxidant capacity of obese rat tissues, and at the same time promotes muscle protein synthesis, improves insulin sensitivity, and promotes glucose metabolism.

\section{Introduction}

Obesity is a chronic metabolic disorder that is growing at an alarming rate, but not all individuals are prone to occur. Numerous studies have shown that under high-fat diet, some animals have a good response to high fat and are prone to obesity, while some animals resist obesity. This phenomenon of Loading [MathJax]/jax/output/CommonHTML/jax.js hibiting different responses to the same high-fat diet is 
defined as obesity-prone (OP) and obesity-resistant (OR) [1]. There were differences in body weight regulation, oxidative stress and energy metabolism between OP and OR rats. Previous studies have found that myoglobin in $\mathrm{OR}$ rats was significantly up-regulated, while tropomyosin $\mathrm{R}$ and troponin I were significantly down-regulated, making $\mathrm{OR}$ rats more effective in regulating muscle contraction, increasing glucose utilization, and maintaining lighter weight [2]. While in OP rats, high-fat diet not only significantly increased body weight, but also decreased mitochondrial membrane potential in the heart and liver, induced mitochondrial DNA damage and dysfunction [3], decreased antioxidant enzyme activity, and leading to oxidative stress [4].

Mitochondria are important sites for energy metabolism. OP and OR individuals have different responses to mitochondrial function. Richard's study found that uncoupling proteins 2 (UCP2) expression was increased 2-fold in white adipose tissue of OR mice compared with OP mice, suggesting that OR mice can increase body heat production and against obesity by uncoupling proteins [5]. High-fat diet can induce a significant increase in mitochondrial copy number in skeletal muscle of OR rats, and increase phosphorylation of AMP-activated protein kinase (AMPK), expression of glucose transporter 4 (GLUT4) and Carnitine palmitoyl transferase 1 (CPT-1) [6], indicating that OR rats have higher mitochondrial activity to resist the energy increase caused by the high fat diet. At present, studies on OP and OR mainly focus on physiological signals and energy metabolism. Mitochondria, as a key factor in energy metabolism, have rarely been reported on mitochondrial function and energy metabolism.

Butyrate, one of short-chain fatty acids (SCFAs), is obtained by fermenting dietary fiber via intestinal microflora and becomes sodium butyrate $(\mathrm{NaB})$ after receiving sodium. It has been demonstrated that $\mathrm{NaB}$ actively involved in a number of pathological processes, including obesity and Type 2 diabetes mellitus (T2DM). Recent studies have found that $5 \% \mathrm{NaB}$ is effective in the treatment of obesity and insulin resistance in the dietary obese model and butyrate activates FGF21 gene transcription by inhibiting HDAC3. Studies also found that $\mathrm{NaB}$ enhanced the activities of glutathione peroxidases (GSH$\mathrm{Px})$, catalase (CAT), manganese superoxide dismutase (Mn-SOD) and reduced the level of ROS[7]. Besides, we have found that $\mathrm{NaB}$ up-regulated the production of ATP. NaB plays a pivotal role in decreasing body weight, enhancing the activity of antioxidant enzymes and promoting metabolism due to the inhibition of HDACs[8-10]. Nevertheless, whether the different dose of NaB in HFD animals is attributed to its antioxidative activity or the functional protection of mitochondrial has not yet been clarified. Thus, we hypotheses that $\mathrm{NaB}$ affecting mitochondrial function may be due to its possible antioxidant effect.

As a fermentation product of intestinal flora, butyric acid has been shown to improve tissue antioxidant enzyme activity, regulate body sugar and lipid metabolism, and have a certain regulatory effect on redox state and mitochondrial metabolism. However, there are no reports on the intervention of sodium butyrate in different obese individuals, and the specific mechanism of action has not been clarified. Therefore, this study induced the obesity prone and obesity resistance model by feeding high-fat diets to SD rats, studied the changes of mitochondrial redox homeostasis and energy metabolism in skeletal muscle and 
pancreas, and explored the possible mechanisms and dose effects of sodium butyrate antioxidant protection.

\section{Materials And Methods}

\section{Animals acquisitions and treatments}

91 male Sprague-Dawley rats (4 weeks old) were obtained from Shanghai Slac Laboratory Animal Co., Ltd. (Shanghai, China). Animals were housed in ventilated cages under standard laboratory conditions with controlled temperature $\left(22^{\circ} \mathrm{C}-26^{\circ} \mathrm{C}\right)$, humidity $(40 \%-70 \%)$ and normal light/dark ( $\left.12 \mathrm{~h} / 12 \mathrm{~h}\right)$ cycle and given access to normal experimental animal diet and water. The protocols were approved by the Ethics Committee of Jiangnan University.

After 1 week acclimation, rats were randomly divided into control group (fed with low-fat diet; $12 \%$ energy from fat; $n=7$ ) and HFD group ( $45 \%$ energy from fat; $n=84)$. At the 8 th week, OP and OR were delineated based on body weight gain (upper 1/3 for OP; lower 1/3 for OR) of the HFD group. Rats in OP and OR groups were fed with high-fat diet supplemented with $4 \%, 5 \%$ and $6 \%$ sodium butyrate respectively. At the 20th week, rats were sacrificed. The blood sample was centrifuged at $3500 \mathrm{~g}$ for $10 \mathrm{~min}$, and the serum was stored at $-80^{\circ} \mathrm{C}$ for further analyses. The tissue $(0.1 \mathrm{~g})$ was homogenized in $0.9 \mathrm{~mL}$ normal saline, and the supernatant was collected through centrifugation at $4000 \mathrm{~g}$ for $10 \mathrm{~min}$. The protein content in the supernatant was measured according to the procedures of an Enhanced BCA Protein Assay Kit (Beyotime Biotech, Shanghai, China). Another part was stored at $-80^{\circ} \mathrm{C}$ for further analyses.

\section{Analysis of ROS and redox state}

ROS was determined by luminol-dependent chemiluminescence assay in the presence of luminal $(0.5 \mathrm{mmol} / \mathrm{L})$ and horseradish peroxidase $(12 \mathrm{U} / \mathrm{mL})$ using a thermostatically $\left(37^{\circ} \mathrm{C}\right)$ controlled luminometer (Xi'an Remex Analysis Instrument, Xi'an, China). Total antioxidant capacity (T-AOC), antioxidant enzymes (SOD, GSH and GSSG) activity and MDA content was measured by appropriate test kits (Jiancheng Bioengineering Institute, Nanjing, China).

\section{Plasma lipid, glucose and insulin status}

Plasma total cholesterol (TC), low-density lipoprotein cholesterol (LDL-C), high-density lipoprotein cholesterol (HDL-C), and triacylglycerol (TG) concentrations were analyzed by the enzymatic colorimetric assay kits (Nanjing Jiancheng Bioengineering Institute, Nanjing, China) according to the instructions of the manufacturer. Blood glucose and insulin were measured by the glucose determination kit (Jiancheng Bioengineering Institute, Nanjing, China) and ELISA kit (Huijia, Xiamen, China). The insulin resistance index (HOMA-IR) is calculated as HOMA-IR = Insulin*glucose/22.5.

\section{Acetyl coenzyme A, NADH/NAD ${ }^{+}$, ATP, the mitochondrial membrane potential, lactic acid content assay}


The concentrations of acetyl coenzyme A, NADH/NAD ${ }^{+}$and ATP, mitochondrial membrane potential, lactic acid in the tissues were measured by ELISA kit (Huijia, Xiamen, China) and ATP, mitochondrial membrane potential, lactic acid determination kit (Beyotime Biotech, Shanghai, China) respectively. The concentration was normalized to that of protein in the same tissue lysates.

\section{Mitochondrial MnSOD and GPX activity assays}

The activities of MnSOD were measured by WST-8 assays after inhibiting the activity of Cu/ZnSOD by a specific inhibitor (Beyotime Biotech, Shanghai, China). Mitochondrial GPX activity was detected according to the manufacturer's instructions (Jiancheng Bioengineering Institute, Nanjing, China).

\section{mtDNA copy number}

In rat tissues, 25 to $50 \mathrm{mg}$ of frozen sample was used for total DNA isolation with manufacturer's instructions (Sangon Biotech, Shanghai, China). The relative mtDNA copy numbers were derived from the ratio of the encoding ribosomal protein $\mathrm{L} 4(\mathrm{Rp} / 4)$ target quantification result to the cytochrome coxidase subunit 1 (COX1) target quantification by real-time PCR as described [11]. The sequences used in this study were shown in Table 1. 
Table 1

Sequences of primers in quantitative real-time PCR

\begin{tabular}{|c|c|c|}
\hline & Forward primer & Reverse primer \\
\hline$\beta$-action & САAССTTCTTGCAGСТССТССT & AGGGTCAGGATGCCTCTCTTGCTC \\
\hline $\mathrm{PI} 3 \mathrm{~K}$ & GTGGGACTGTGACCGAAAGT & GCTTAGGGCTGGTTCTCCTT \\
\hline GSK-3 $\beta$ & САТССТТАТСССТССТСАСG & AGAAGCGGCGTTATTGGTCT \\
\hline $\mathrm{HO}-1$ & CAGAAGGGTCAGGTGTCCAG & GAAGGCCATGTCCTGCTCTA \\
\hline NQO-1 & ACATCACAGGGGAGCCGAAGGACT & GGCACCCCAAACCAATACAATG \\
\hline Nrf2 & GAGACGGCCATGACTGAT & GTGAGGGGATCGATGAGTAA \\
\hline TFAM & CAGAGTTGTCATTGGGATTGG & TTCAGTGGGCAGAAGTCCAT \\
\hline PGC-1a & GGCCGGAGCAATCTGAGTTA & GGCCGTTTAGTCTTCCTTTCCT \\
\hline IRS-1 & CTGGACGTCACAGGCAGAAT & CGTGAGGTCCTGGTTGTGAA \\
\hline GLUT4 & GTTGGTCTCGGTGCTCTTAG & GGCCACGATGGACACATAAC \\
\hline mTOR & GCAGATTTGCCAACTACC & TCTCCGGCCCTCATTTCG \\
\hline S6K1 & CGTGGAGTCTGCGGCGGGTC & CGCTCTGCTTTCGTGTGGGC \\
\hline Myogenin & GGAGTCCAGAGAGCCCCCTTGTTAA & CGGTCGGGGCACTCACTGTCTCT \\
\hline Myostain & CCATGCCTACAGAGTCTGAC & CTTCTAAAAAGGGATTCAGCC \\
\hline Bax & CATGAAGACAGGGGCCTTTTTG & TCAGCTTCTTGGTGGATGCGTC \\
\hline Caspase-3 & GGATTACCCTGAAATGGGCTTGT & CTCTGAGGTTAGCTGCATCGACAT \\
\hline $\mathrm{Bcl}-2$ & АCTTCTCTCGTCGCTACCGTCG & CCTGAAGAGTTCCTCCACCACC \\
\hline
\end{tabular}

Total RNA in liver, gastrocnemius muscle and pancreas were extracted using the Trizol reagent (Generay, Shanghai, China). Total RNA (1000 ng/ $\mu \mathrm{L} ; 260: 280=1.8 \sim 2.0)$ was reverse-transcribed to CDNA using High-Capacity cDNA Reverse Transcription Kit with RNase Inhibitor (Thermo Fisher Scientific, Massachusetts, USA). For quantification, real-time PCR analysis was performed using QuantiTect SYBR Green PCR Kits on ABI 7500 Fast Real Time PCR. $\beta$-actin expression was used as an endogenous control. The sequences of the primers used are listed in Table 1.

\section{Western blotting analysis}

Tissues were homogenized in cooled RIPA buffer (Jiancheng Bioengineering Institute, Nanjing, China) and centrifuged at $15,000 \mathrm{~g}$ for $10 \mathrm{~min}$ at $4{ }^{\circ} \mathrm{C}$. The supernatant proteins were collected and the connantratinn wne datarminnd wainnthn RrA protein assay kit (Beyotime). Equal amount of tissueLoading [MathJax]/jax/output/CommonHTML/jax.js 
denatured proteins in loading buffer of each sample were loaded onto the sodium dodecylsulphatepolyacrylamide gel and electrical separated. The target proteins were transformed onto PVDF membranes (Millipore, Billerica, MA, USA) using wet transfer (Bio-Rad) and blocking with $5 \%$ bovine serum albumin for $1 \mathrm{~h}$. The membranes were incubated with primary antibodies at $4{ }^{\circ} \mathrm{C}$ overnight. The primary antibodies-binding membranes were washed with Tris Buffered Saline with Tween-20 (TBST) and incubated with HRP-conjugated secondary antibody for $1 \mathrm{~h}$ and then washed with TBST again. The blots were then visualized using enhanced chemiluminescence solution (Bio-Rad). Relative protein expression levels were standardized with $\beta$-actin and normalized with the control group.

\section{Statistical analysis}

Experimental data are presented as means \pm standard error. The differences between groups were analyzed by one-way ANOVA test with Tukey's HSD test at the significant level of 0.05 and 0.01 . All statistical analyses were performed with SPSS Statistics ver. 19.0.

\section{Results}

\section{Effect of NaB Intervention on body weight and insulin resistant index}

Figure 1 showed changes in the body weight, insulin, blood glucose and insulin resistant index after 20 weeks. Compared with the control group, HFD significantly increased body weight, blood glucose, insulin and HOMA-IR of rats in the OP group $(P<0.05)$, whereas there was no difference in the OR group. The body weight, insulin and insulin resistance index of the rats in the OR group were significantly lower than those in the OP group $(P<0.05)$. Figure 1 a showed that $6 \% \mathrm{NaB}$ can significantly reduce the body weight of rats in the OP group, while $4 \%, 5 \%, 6 \%$ can significantly reduce the body weight in the OR group. Different doses of $\mathrm{NaB}$ had different effects on blood glucose and insulin in rats, $4 \%, 5 \%, 6 \% \mathrm{NaB}$ can significantly reduce the insulin resistance index of rats in the OP group, while $6 \% \mathrm{NaB}$ had no effect on in the OR group.

\section{Effect of $\mathrm{NaB}$ Intervention on Plasma lipid}

As shown in Table 2, TG, TC, LDL-C and FFA were significantly higher in OP rats compared with Con rats $(P<0.05)$. Sodium butyrate intervention can improve blood lipid levels, the effect of $6 \%$ sodium butyrate intervention was more significant $(P<0.05)$. However, in high-fat diet fed OR rats, except for FFA, which was significantly higher than the Con group $(P<0.05)$, there was no significant difference in other indicators of Con, indicating that OR rats can maintain good blood lipid levels. Different doses of sodium butyrate intervention can significantly reduce the FFA level and increase the HDL-C content in OR rats $(P<$ 0.05 ), and $4 \%$ sodium butyrate can completely return to normal levels after intervention. 
Table 2

Effect of NaB on plasma lipid status of HFD fed SD rats

\begin{tabular}{|c|c|c|c|c|c|}
\hline & $\begin{array}{l}\text { FFA } \\
\left(10^{3} \mu \mathrm{mol} / \mathrm{L}\right)\end{array}$ & $\begin{array}{l}\text { TG } \\
(\mu \mathrm{mol} / \mathrm{mL})\end{array}$ & $\begin{array}{l}\text { TC } \\
(\mu \mathrm{mol} / \mathrm{mL})\end{array}$ & $\begin{array}{l}\text { HDL-C } \\
(\mu \mathrm{mol} / \mathrm{mL})\end{array}$ & $\begin{array}{l}\text { LDL-C } \\
(\mu \mathrm{mol} / \mathrm{mL})\end{array}$ \\
\hline Con & $1.17 \pm 0.07$ & $0.93 \pm 0.03$ & $1.13 \pm 0.11$ & $0.61 \pm 0.06$ & $0.14 \pm 0.01$ \\
\hline $\mathrm{OP}$ & $1.74 \pm 0.11 * \mathrm{C}$ & $1.21 \pm 0.06^{* \mathrm{~B}}$ & $1.42 \pm 0.07 \star B$ & $0.60 \pm 0.03^{B}$ & $0.20 \pm 0.01 *$ \\
\hline $\mathrm{OP}+4 \% \mathrm{NaB}$ & $0.91 \pm 0.07^{\mathrm{B}}$ & $1.04 \pm 0.03^{\mathrm{AB}}$ & $1.25 \pm 0.06^{\mathrm{AB}}$ & $0.61 \pm 0.02^{\mathrm{B}}$ & $0.16 \pm 0.01$ \\
\hline $\begin{array}{l}\mathrm{OP}+5 \% \mathrm{NaB} \\
\mathrm{OP}+6 \% \mathrm{NaB}\end{array}$ & $1.03 \pm 0.05^{\mathrm{B}}$ & $1.01 \pm 0.10^{\mathrm{AB}}$ & $1.31 \pm 0.07^{A B}$ & $0.65 \pm 0.03^{A B}$ & $\begin{array}{l}0.18 \pm 0.02 \\
0.16 \pm 0.01\end{array}$ \\
\hline $\mathrm{OR}$ & $0.58 \pm 0.03^{A}$ & $0.82 \pm 0.04^{A}$ & $1.07 \pm 0.07^{A}$ & $0.57 \pm 0.03^{B}$ & $0.15 \pm 0.01$ \\
\hline $\mathrm{OR}+4 \% \mathrm{NaB}$ & $1.33 \pm 0.15^{\star b}$ & $0.94 \pm 0.08^{\#}$ & $1.15 \pm 0.05^{\#}$ & $0.63 \pm 0.05^{a}$ & $0.16 \pm 0.01$ \\
\hline $\mathrm{OR}+5 \% \mathrm{NaB}$ & $0.62 \pm 0.04^{a}$ & $0.85 \pm 0.06$ & $1.10 \pm 0.05$ & $0.84 \pm 0.04^{b}$ & $0.15 \pm 0.01$ \\
\hline \multirow[t]{2}{*}{$\mathrm{OR}+6 \% \mathrm{NaB}$} & $0.7 \pm 0.08^{a}$ & $0.88 \pm 0.08$ & $1.11 \pm 0.09$ & $0.80 \pm 0.06^{b}$ & $0.17 \pm 0.03$ \\
\hline & $0.83 \pm 0.07^{a}$ & $0.85 \pm 0.07$ & $1.11 \pm 0.07$ & $0.82 \pm 0.03^{b}$ & \\
\hline
\end{tabular}

\section{Effect of NaB Intervention on Redox state}

The markers of tissue oxidative stress and antioxidant enzymes were shown in Table 3. HFD caused a decrease in total antioxidant capacity and T-SOD content in the gastrocnemius of OP rats. The oxidative stress of the pancreas was more serious than that of the gastrocnemius muscle, which showed that the total antioxidant capacity decreases, GSH/GSSG and T-SOD decrease, and MDA and ROS content increase, indicating an impairment of antioxidant system in the liver, gastrocnemius and pancreas. However, $\mathrm{OP}+6 \%$ and $\mathrm{OR}+4 \%$ groups exhibited an obvious increase of the activity of antioxidant enzymes T-AOC, GSH/ GSSG and SOD, with the down-regulation of MDA and ROS. These data suggested that antioxidant capacity of gastrocnemius muscle and pancreas tissues was restored. 
Table 3

Effect of $\mathrm{NaB}$ on antioxidant index of HFD fed SD rats

T-AOC

$\mathrm{U} / \mathrm{mg}$ prot
MDA

$\mathrm{nmol} / \mathrm{mg}$

prot
ROS

$1.00 \pm 0.14$

$1.21 \pm$

$0.12^{* b}$

$1.93 \pm 0.51^{b}$

$0.12 * 6$

$\mathrm{OP}+4 \% \mathrm{NaB}$

$0.48 \pm$

$0.03^{\mathrm{ab}}$

$1.64 \pm$

$0.28^{\mathrm{ab}}$

$\mathrm{OP}+5 \% \mathrm{NaB}$

$0.54 \pm$

$0.06^{\mathrm{b}}$

$1.43 \pm 0.32^{\mathrm{a}}$

$1.00 \pm 0.07^{a}$

$6.81 \pm 0.34^{b}$

$6.32 \pm 0.46$

$653.36 \pm 22.77$

$\mathrm{U} / \mathrm{mg}$ prot

T-SOD

gastrocnemius

muscle

\begin{tabular}{|c|c|c|c|c|c|}
\hline $\mathrm{OP}+5 \% \mathrm{NaB}$ & $\begin{array}{l}0.54 \pm \\
0.06^{\mathrm{b}}\end{array}$ & $1.43 \pm 0.32^{\mathrm{a}}$ & $0.88 \pm 0.08^{a}$ & $7.03 \pm 0.35^{b}$ & $\begin{array}{l}564.83 \pm \\
20.34^{\mathrm{b}}\end{array}$ \\
\hline $\mathrm{OP}+6 \% \mathrm{NaB}$ & $\begin{array}{l}0.52 \pm \\
0.05^{b}\end{array}$ & $1.42 \pm 0.16^{a}$ & $0.78 \pm 0.12^{\mathrm{a}}$ & $7.58 \pm 0.54^{b}$ & $\begin{array}{l}554.32 \pm \\
10.68^{b}\end{array}$ \\
\hline OR & $\begin{array}{l}0.41 \pm \\
0.03^{\# A}\end{array}$ & $1.62 \pm 0.13^{B}$ & $\begin{array}{l}0.94 \pm \\
0.10^{\# B}\end{array}$ & $\begin{array}{l}5.90 \pm \\
0.31^{\mathrm{A}}\end{array}$ & $\begin{array}{l}527.73 \pm \\
39.55^{\star A}\end{array}$ \\
\hline $\mathrm{OR}+4 \% \mathrm{NaB}$ & $\begin{array}{l}0.53 \pm \\
0.06^{\mathrm{B}}\end{array}$ & $1.34 \pm 0.23^{A}$ & $\begin{array}{l}0.85 \pm \\
0.08^{A B}\end{array}$ & $7.28 \pm 0.24^{B}$ & $\begin{array}{l}587.44 \pm \\
21.45^{\mathrm{B}}\end{array}$ \\
\hline $\mathrm{OR}+5 \% \mathrm{NaB}$ & $\begin{array}{l}0.46 \pm \\
0.02^{A B}\end{array}$ & $1.38 \pm 0.18^{A}$ & $\begin{array}{l}0.89 \pm \\
0.07^{A B}\end{array}$ & $7.52 \pm 0.14^{B}$ & $\begin{array}{l}603.24 \pm \\
34.62^{\mathrm{B}}\end{array}$ \\
\hline $\mathrm{OR}+6 \% \mathrm{NaB}$ & $\begin{array}{l}0.47 \pm \\
0.02^{\mathrm{AB}}\end{array}$ & $\begin{array}{l}1.54 \pm \\
0.22^{\mathrm{AB}}\end{array}$ & $\begin{array}{l}0.79 \pm \\
0.09^{A}\end{array}$ & $\begin{array}{l}6.57 \pm \\
0.61^{A B}\end{array}$ & $\begin{array}{l}568.43 \pm \\
24.56^{\mathrm{AB}}\end{array}$ \\
\hline \multicolumn{6}{|l|}{ pancreas } \\
\hline Con & $1.10 \pm 0.08$ & $0.96 \pm 0.08$ & $1.00 \pm 0.12$ & $5.99 \pm 0.32$ & $416.42 \pm 9.12$ \\
\hline OP & $\begin{array}{l}0.76 \pm \\
0.03^{\star a}\end{array}$ & $\begin{array}{l}1.45 \pm \\
0.16^{\star b}\end{array}$ & $\begin{array}{l}1.24 \pm \\
0.10 * b\end{array}$ & $\begin{array}{l}5.00 \pm \\
0.16^{\star a}\end{array}$ & $\begin{array}{l}231.39 \pm \\
14.44^{* a}\end{array}$ \\
\hline $\mathrm{OP}+4 \% \mathrm{NaB}$ & $\begin{array}{l}0.99 \pm \\
0.11^{\mathrm{ab}}\end{array}$ & $\begin{array}{l}1.25 \pm \\
0.20^{\mathrm{ab}}\end{array}$ & $\begin{array}{l}1.13 \pm \\
0.12^{\mathrm{ab}}\end{array}$ & $\begin{array}{l}5.63 \pm \\
0.50^{\mathrm{ab}}\end{array}$ & $\begin{array}{l}233.17 \pm \\
32.98^{a}\end{array}$ \\
\hline $\mathrm{OP}+5 \% \mathrm{NaB}$ & $\begin{array}{l}0.93 \pm \\
0.18^{\mathrm{ab}}\end{array}$ & $0.90 \pm 0.11^{a}$ & $\begin{array}{l}1.17 \pm \\
0.11^{\mathrm{ab}}\end{array}$ & $\begin{array}{l}5.78 \pm \\
0.35^{\mathrm{ab}}\end{array}$ & $\begin{array}{l}321.90 \pm \\
32.43^{b}\end{array}$ \\
\hline
\end{tabular}

Values are expressed as mean $\pm S E, n=7$. OP, obesity-prone; OR, obesity-resistant; MDA:

Malondialdehyde; ROS, reactive oxygen species; SOD, superoxide dismutase. ${ }^{*} P<0.05$ versus the control group, \# $\mathrm{P}<0.05$ versus the OP qroup. Different letters indicate significant differences, $\mathrm{P}<$ Loading [MathJax]/jax/output/CommonHTML/jax.js 


\begin{tabular}{|c|c|c|c|c|c|}
\hline & $\begin{array}{l}\text { T-AOC } \\
\text { U/mg prot }\end{array}$ & $\begin{array}{l}\text { MDA } \\
\text { nmol/mg } \\
\text { prot }\end{array}$ & ROS & GSH/GSSG & $\begin{array}{l}\text { T-SOD } \\
\text { U/mg prot }\end{array}$ \\
\hline $\mathrm{OP}+6 \% \mathrm{NaB}$ & $\begin{array}{l}1.14 \pm \\
0.16^{\mathrm{b}}\end{array}$ & $1.06 \pm 0.14^{a}$ & $0.99 \pm 0.12^{a}$ & $6.83 \pm 0.33^{b}$ & $\begin{array}{l}373.78 \pm \\
34.28^{\mathrm{b}}\end{array}$ \\
\hline OR & $\begin{array}{l}0.98 \pm \\
0.04^{\star A}\end{array}$ & $\begin{array}{l}1.22 \pm \\
0.15^{\star C}\end{array}$ & $\begin{array}{l}1.18 \pm \\
0.15^{\star B}\end{array}$ & $\begin{array}{l}5.15 \pm \\
0.27^{\star A}\end{array}$ & $\begin{array}{l}266.80 \pm \\
14.49 * A\end{array}$ \\
\hline $\mathrm{OR}+4 \% \mathrm{NaB}$ & $\begin{array}{l}1.32 \pm \\
0.11^{\mathrm{B}}\end{array}$ & $0.75 \pm 0.09^{A}$ & $\begin{array}{l}1.01 \pm \\
0.20^{\mathrm{AB}}\end{array}$ & $7.30 \pm 0.32^{\mathrm{B}}$ & $\begin{array}{l}354.79 \pm \\
37.57^{B}\end{array}$ \\
\hline $\mathrm{OR}+5 \% \mathrm{NaB}$ & $\begin{array}{l}1.43 \pm \\
0.11^{\mathrm{B}}\end{array}$ & $\begin{array}{l}0.92 \pm \\
0.03^{A B}\end{array}$ & $\begin{array}{l}0.92 \pm \\
0.15^{A}\end{array}$ & $7.47 \pm 0.19^{B}$ & $\begin{array}{l}372.54 \pm \\
13.38^{\mathrm{B}}\end{array}$ \\
\hline $\mathrm{OR}+6 \% \mathrm{NaB}$ & $\begin{array}{l}1.20 \pm \\
0.08^{\mathrm{AB}}\end{array}$ & $1.15 \pm 0.07^{C}$ & $\begin{array}{l}1.03 \pm \\
0.08^{\mathrm{AB}}\end{array}$ & $\begin{array}{l}6.35 \pm \\
0.59^{A B}\end{array}$ & $\begin{array}{l}300.19 \pm \\
19.01^{A B}\end{array}$ \\
\hline
\end{tabular}

\section{Effect of $\mathrm{NaB}$ Intervention on the expression of genes and proteins in redox state}

Antioxidant relative genes and proteins in OP and OR groups are shown in Fig. 2. The levels of PI3K, Nrf2 and downstream antioxidant enzymes NQO-1 and HO-1 in gastrocnemius muscle in OP and OR groups were significantly decreased, and GSK-3 $\beta$ was significantly increased compared with Con group $(P<$ 0.05). NaB significantly up-regulated PI3K and down-regulated GSK-3 $\beta$ expression. Otherwise, $\mathrm{NaB}$ significantly up-regulate the expression of Nrf2 and downstream antioxidant enzymes NQO-1 and HO-1 in OP and OR groups. However, different doses had different effects on OP and OR rats, OP group showed better effect in $6 \% \mathrm{NaB}$, and OR group showed better effect in $4 \% \mathrm{NaB}$.

\section{Effect of $\mathrm{NaB}$ Intervention on Mitochondrial functions in gastrocnemius muscle}

As shown in Fig. 3. Compared with the control group, the high-fat diet significantly decreased Mn-SOD, GSH-Px activity, ATP content, NADH/NAD ${ }^{+}$ratio and PGC-1 a expression, increased lactic acid level in OP and OR rats' gastrointestinal muscles. Compared with the Con group, the gastrocnemius mitochondrial membrane potential of the OP group rats decreased significantly, and the OR group rats showed a downward trend. Meanwhile, high-fat diet significantly decreased the mtDNA copy number and the expression of TFAM in OP rats, but there was no significant effect on OR rats. However, $\mathrm{NaB}$ ( $6 \%$ for OP rats, $4 \%$ for OR rats) treatment significantly increased the expression of PGC- $1 \mathrm{a}$ and TFAM, increase the antivity of antinvidant anzvmac (faCH_Dv and Mn-SOD) in mitochondria, increase the production of ATP, Loading [MathJax]/jax/output/CommonHTML/jax.js 
acetyl-CoA and NADH/NAD ${ }^{+}$ratio, reduce the lactic acid content in muscle, and increase the mitochondrial membrane potential and mRNA copy number, which reduces oxidative stress caused by high-fat diets and enhances energy metabolism.

\section{Effect of $\mathrm{NaB}$ Intervention on Gastrocnemius muscle synthesis and insulin sensitivity}

The effect of $\mathrm{NaB}$ intervention on muscle synthesis and metabolism in OP and OR rats is shown in Fig. 4 $(a, b, c, d)$. Compared with the Con group, high-fat diet up-regulated the expression of Mtor, S6k1 and myostain mRNA in SD rats, in OR group the expression of myogenin was down-regulated, and the expression of Mtor and S6k1 were up-regulated. After 12 weeks of $\mathrm{NaB}$ intervention, the above indicators were significantly improved, indicating that $\mathrm{NaB}$ can improve muscle synthesis, promote muscle deposition, and reduce the risk of obesity, of which $\mathrm{OP}+6 \% \mathrm{NaB}, \mathrm{OR}+4 \% \mathrm{NaB}$ effect is more significant.

Compared with the Con group, the expression of Ampk and Sirt1 mRNA in the OP group and the expression of Sirt1 mRNA in the OR group were significantly down-regulated $(P<0.05)$. After $\mathrm{NaB}$ intervention, the expression of Ampk and Sirt1 were significantly up-regulated, indicating that $\mathrm{NaB}$ can promote the utilization of sugar by gastrocnemius muscle by affecting the expression of Ampk and Sirt1.

After that, we measured the insulin receptor and glucose transport related genes in rats. As shown in Fig. 4 (g, h). Compared with the control group, the expressions of Irs-1 and Glut 4 mRNA in the OP group were significantly down-regulated, but there was no significant difference between OR and control group. $\mathrm{NaB}$ intervention significantly up-regulated the mRNA expression of Irs-1 and Glut 4 for 12 weeks, which had an important regulatory effect on increasing insulin sensitivity and promoting glucose utilization.

\section{Effect of $\mathrm{NaB}$ Intervention on insulin-related gene expression}

Insulin secretion relative genes are shown in Fig. $5(a, b)$. Relative mRNA levels of Pdx-1, Mafa were significantly down-regulated in both OP and OR groups compared with control group. Meanwhile, the expression of genes above were elevated with $\mathrm{NaB}$ intervention in OP and OR groups. $5 \%$ and $6 \% \mathrm{NaB}$ significantly increased the expression of $\mathrm{Pdx}-1$ in OP rats, while OR rats were more effective at $4 \%$ and $5 \%$. NaB up-regulated the expression of Mafa in OP and OR rats, and there was no significant difference among three doses. The results indicated that $\mathrm{NaB}$ intervention can increase insulin synthesis.

Islet cell apoptosis related gene expression as shown in Fig. 5 (c, d, e). Compared with Con group, the mRNA expression of anti-apoptosis factor Bcl-2 was significantly down-regulated, the mRNA expressions of pro-apoptotic factors Bax and Caspase 3 were significantly up-regulated in OP group and OR group, indicating that high-fat diet can cause islet cell apoptosis in obese rats. In this study, $\mathrm{NaB}$ intervention significantly up-regulated the expression of $\mathrm{Bcl}-2$ mRNA levels in the pancreas of OP and OR rats, and down-regulated the mRNA expression of Bax and Caspase 3 mRNA, OP $+6 \% \mathrm{NaB}$ and $\mathrm{OR}+4 \% \mathrm{NaB}$ showed better effects.

Loading [MathJax]/jax/output/CommonHTML/jax.js

Page $11 / 21$ 


\section{Discussion}

Previous studies have shown that butyric acid or its pre-derivatives has beneficial effects on glucose homeostasis and body weight maintenance[3, 12]. However, few of them consider the effect of sodium butyrate on skeletal muscle mitochondria and different fat phenotypes. In the present study, we confirmed that $\mathrm{NaB}$ has the similar effect as previous studies did, $\mathrm{NaB}$ intervention could effectively reduce the body weight of rats in the OP and OR group, and reduce insulin resistance, plasma lipid. Moreover, our study showed that the regulation of $\mathrm{NaB}$ significantly increases the activity of mitochondrial antioxidant enzymes MnSOD and GSH-Px, mitochondrial membrane potential, $\mathrm{NADH} / \mathrm{NAD}^{+}$ratio, acetyl-CoA and ATP production, TFAM and PGC-1a expression, and mitochondrial DNA copy number. $6 \% \mathrm{NaB}$ intervention in $\mathrm{OP}$ rats, $4 \% \mathrm{NaB}$ intervention in $\mathrm{OR}$ rats was more significant, indicating that there is dose effect of $\mathrm{NaB}$ intervention in different obesity phenotypes.

Oxidative stress in HFD-fed rats was presented as higher plasma ROS and lower plasma antioxidant levels in accordance with previous studies[13, 14]. In the present study, the results indicated that OP and $\mathrm{OR}$ rats showed different degrees of oxidative damage. $\mathrm{NaB}$ intervention mitigated HFD-induced oxidative damages (ROS, MDA) and increased antioxidant capacity biomarkers (SOD, GSH/GSSG and T-AOC) in gastrocnemius muscle and pancreatic. $4 \%$ and $6 \% \mathrm{NaB}$ intervention showed better effect in OR and OP groups respectively. Nrf2-ARE signaling is the main antioxidant pathway in vivo. Nrf2, as a key antioxidant factor, can regulate downstream NQO-1, HO-1 and other antioxidant enzymes transcription and expression[15]. PI3K and GSK-3 $\beta$ could regulate the proportion of Nrf2 into the nucleus, activate PI3K or down-regulated the expression of GSK-3 $\beta$ in order to enhance Nrf2 entry [16]. Oxidative stress induced by high-fat diet is closely related to Nrf2-ARE pathway, in this study, the results showed that NaB significantly up-regulated PI3K and down-regulated GSK-3 $\beta$ expression, indicating that the mechanism $\mathrm{NaB}$ protects against oxidative stress may relate to the regulation of Nrf2-ARE signaling pathway. Meanwhile, these data showed that $6 \% \mathrm{NaB}$ and $4 \% \mathrm{NaB}$ had significant effects on the expression of Nrf2-ARE pathway-related genes in OP and OR rats, indicating that there is a dose-effect $\mathrm{NaB}$ antioxidant protection.

Mitochondrial function shows an crucial impact on whole-body metabolism. As important antioxidant enzymes in mitochondria, Mn-SOD and GSH-Px are essential for the maintenance of mitochondrial redox homeostasis. Acetyl-CoA and NADH/NAD ${ }^{+}$ratios are the major indicators of mitochondrial energy metabolism. In our study, $\mathrm{NaB}$ intervention could restore the above indicators in obesity rats. Our previous study found that $\mathrm{H}_{2} \mathrm{O}_{2}$ induced oxidative damage in HepG2 cells, and reduced mitochondrial acetyl CoA content, membrane potential and ATP production. Resveratrol can restore mitochondrial metabolism by increasing the levels of Mn-SOD and GSH-Px in AF mice[17]. Our data also show that NaB can improve mitochondrial function, increase mitochondrial membrane potential, increase ATP production, and reduce lactic acid accumulation in gastrocnemius muscle by increasing the levels of Mn-SOD and GSH-Px. In addition to mitochondrial dysfunction, we also found that mitochondrial mRNA copy number decreased in OP and OR rats. It has been reported that PGC1 $a$ and TFAM regulate mitochondrial copy number[18, 
mitochondrial copy number in OP rats. Recent study showed that accumulation of TFAM protein could increase the expressions of mtDNA in skeletal muscle, indicating that $\mathrm{NaB}$ may enhance mitochondrial biogenesis by enhancing the PGC1a and TFAM expression in rats.

Mechanistic Target of Rapamycin (mTOR) signaling pathway plays an important role in protein synthesis and cell growth. mTOR participates in cell differentiation, proliferation and protein synthesis by activating the downstream protein S6K1[20]. Previous studies have shown that skeletal muscle mTOR levels and downstream S6K1 phosphorylation levels increase after exercise, and protein synthesis rate increases. Myostain, a member of the transforming growth factor-beta superfamily of secreted growth and differentiation factors, plays an important role in muscle development [21]. In mice knocking out the myostain gene, skeletal muscle weight was significantly increased, and excessive muscle growth was also observed in mice with decreased myostain expression. The myogenin gene is a myogenic determinant and necessary factor for skeletal muscle differentiation [22]. Previous studies have shown that the risk of obesity in C57BL/6J mice may be related to the over-activation of mTOR-S6K1 signaling pathway and decreased AKT levels[23], which is consistent with the results of this study. In our study, we found that $\mathrm{OP}+6 \%$ and $\mathrm{OR}+4 \% \mathrm{NaB}$ significantly increased the expression of muscle and related genes, which may be an important factor in $\mathrm{NaB}$ intervention to reduce body weight.

SIRT1 is a nicotinamide adenine dinucleotide $\left(\mathrm{NAD}^{+}\right)$-dependent deacetylase. In mice lacking SIRT1 deacetylase activity, skeletal muscle insulin signaling is diminished and insulin resistance occurs [24]. The AMPK signaling pathway is the central link regulating cell energy status. It activates and promotes phosphorylation of downstream signaling molecules [25]. Peng [26] found that NaB treatment of colon increased AMPK activity, consistent with the results of this study. SIRT1 and AMPK are intracellular energy receptors that regulate the activity of PGC-1a by phosphorylation and deacetylation.

Previous studies found that Glut 4 mRNA levels in skeletal muscle of type 2 diabetic mice was significantly decreased, spironolactone can significantly increase the expression of Glut 4 and improve glucose utilization[27]. Compared with the control group, the expression of IRS-1 mRNA in skeletal muscle of diabetic rats was significantly decreased, and chromium supplementation up-regulated the expression of IRS-1 mRNA and increased insulin resistance. Our study found that NaB intervention for 12 weeks can significantly increase the expression of IRS- 1 and Glut 4 mRNA, and OP $+6 \% \mathrm{NaB}, \mathrm{OR}+4 \% \mathrm{NaB}$ has a significant effect on the improvement of insulin sensitivity and promote glucose intake.

Pancreatic redox homeostasis is closely related to insulin secretion. High-fat diet induced imbalanced redox homeostasis in obese mice, reduced pancreatic dysfunction and insulin secretion. The expression of Pdx 1 and MafA in obese mice induced by high fat decreased accomponied with dysfunction of pancreas [28]. This study found that $\mathrm{NaB}$ intervention could increase the expression of Pdx 1 and MafA mRNA and insulin secretion in the pancreas.

Consistent with previous publications, our results showed that the expression of $\mathrm{Bcl}-2$ were significantly down-regulated after a HFD with the increase of Bax and Caspase 3 mRNA levels. Qi et al [29] showed 
that radiation significantly reduced the expression of Bcl-2 mRNA, increased the expression of Bax and Caspase 3, and excessive oxidative stress altered the expression level of apoptosis-related genes. The study found that Schisandra oil can significantly reduce the content of MDA in pancreatic tissue of diabetic rats, up-regulate the levels of SOD and CAT and increase the level of Bcl-2 protein, indicating that the improvement of oxidative stress can inhibit apoptosis[30]. In this study, NaB intervention significantly improved pancreatic cell function, improve oxidative stress, and inhibit pancreatic cell apoptosis, and OP $+6 \% \mathrm{NaB}$ and $\mathrm{OR}+4 \% \mathrm{NaB}$ had significant effects. Based on the above findings, we found that $\mathrm{NaB}$ regulates the main pathways of blood glucose homeostasis in OP and OR rats: on the one hand, downregulates the expression of Bax and Caspase 3 , up-regulates Bcl-2 expression, inhibits pancreatic cell apoptosis; On the one hand, the expression levels of $\mathrm{Pdx} 1$ and Mafa are up-regulated and insulin secretion is promoted; in addition, the expression of IRS-1 and GLUT4 mRNA is increased, muscle synthesis is promoted, gastrocnemius insulin sensitivity is increased, and blood glucose and insulin resistance are controlled.

\section{Conclusions}

Our study found that in high-fat diet-induced obese SD rats, the addition of NaB improves oxidative stress in the gastrocnemius and pancreas, thereby improving mitochondrial function, increasing insulin secretion and muscle insulin sensitivity. In addition, the optimal intervention doses of different obesity phenotypes for $\mathrm{NaB}$ were different, $6 \%$ intervention in OP rats, and $4 \%$ intervention in OR rats were more effective, and the relevant mechanism needs further study.

\section{Abbreviations}

$\mathrm{NaB}$

Sodium butyrate; OP:obesity-prone; OR:obesity-resistant; UCP2:uncoupling proteins 2; AMPK:AMPactivated protein kinase; GLUT4:glucose transporter 4; CPT-1:Carnitine palmitoyl transferase 1; SCFAs:short-chain fatty acids; T2DM:Type 2 diabetes mellitus; GSH-Px:glutathione peroxidases; CAT:catalase; Mn-SOD:manganese superoxide dismutase; TC:total cholesterol; LDL-C:low-density lipoprotein cholesterol; HDL-C:high-density lipoprotein cholesterol; TG:triacylglycerol.

\section{Declarations}

\section{Ethics approval and consent to participate}

The animal protocol was approved by the institutional Animal Care and Use Committee at the Jiangnan University.

\section{Consent for publication}


Availability of data and material

The datasets used and/or analysed during the current study are available from the corresponding author on reasonable request.

\section{Competing interests}

The authors have declared that no conflict of interest exists.

\section{Funding}

The study was supported by the National Natural Science Foundation of China (No. 31901679), Wuxi Municipal Science and Education Strengthening Health Engineering Medical Key Discipline Construction Program (ZDXK003) and Young Talent Project (QNRC039).

\section{Author Contributions}

Conceptualization, X.T. and R.Q.Y; Investigation, Y.J.S., Y.R.L and S.H.M..; Formal Analysis, Y.P.L. and Y.J.S.; Writing-Original Draft Preparation, Y.P.L. and Y.J.S.; Writing-Review \& Editing, S.H.M. K.Z.

\section{Acknowledgements}

None.

\section{References}

1. InSurk J: Physiological difference between dietary obesity-susceptible and obesity-resistant Sprague Dawley rats in response to moderate high fat diet. . Experimental animals 2003, 2.

2. Dong Hyun K: Changes in expression of skeletal muscle proteins between obesity-prone and obesityresistant rats induced by a high-fat diet. . Journal of proteome research 2011, 3 .

3. Khan S, Jena G: Sodium butyrate reduces insulin-resistance, fat accumulation and dyslipidemia in type-2 diabetic rat: A comparative study with metformin. Chemico-Biological Interactions 2016, 254:124-134.

4. Yu H-T, Fu X-Y, Liang B, Wang S, Liu J-K, Wang S-R, Feng Z-H: Oxidative damage of mitochondrial respiratory chain in different organs of a rat model of diet-induced obesity. European Journal of Nutrition 2018, 57:1957-1967.

5. R S S: Diet-induced changes in uncoupling proteins in obesity-prone and obesity-resistant strains of mice. . Proceedings of the National Academy of Sciences of the United States of America 1998, 7. 
6. Sun J, Huang T, Qi Z, You S, Dong J, Zhang C, Qin L, Zhou Y, Ding S: Early Mitochondrial Adaptations in Skeletal Muscle to Obesity and Obesity Resistance Differentially Regulated by High-Fat Diet. Exp Clin Endocrinol Diabetes 2017, 125:538-546.

7. Walsh ME, Bhattacharya A, Sataranatarajan K, Qaisar R, Sloane L, Rahman MM, Kinter M, Remmen HV: The histone deacetylase inhibitor butyrate improves metabolism and reduces muscle atrophy during aging. Aging Cell 2015, 14:957.

8. Aguilar EC, Da JS, Naviapelaez JM, Leonel AJ, Lopes LG, Menezesgarcia Z, Ferreira A, Capettini L, Teixeira LG, Lemos VS: Sodium butyrate modulates adipocyte expansion, adipogenesis, and insulin receptor signaling by upregulation of PPAR- $y$ in obese Apo E knockout mice. Nutrition 2018, 47:7582.

9. Valvassori SS, Dalpont GC, Steckert AV, Varela RB, Lopesborges J, Mariot E, Resende WR, Arent CO, Carvalho AF, Quevedo J: Sodium butyrate has an antimanic effect and protects the brain against oxidative stress in an animal model of mania induced by ouabain. Psychiatry Research 2015, 235:154-159.

10. Gao Z, Yin J, Zhang J, Ward RE, Martin RJ, Lefevre M, Cefalu WT, Ye J: Butyrate improves insulin sensitivity and increases energy expenditure in mice. Diabetes 2009, 58:1509-1517.

11. Snowdin JW, Hsiung CH, Kesterson DG, Kamath VG, McKee EE: Effects of Zidovudine Treatment on Heart mRNA Expression and Mitochondrial DNA Copy Number Associated with Alterations in Deoxynucleoside Triphosphate Composition in a Neonatal Rat Model. Antimicrob Agents Chemother 2015, 59:6328-6336.

12. Zhanguo G: Butyrate improves insulin sensitivity and increases energy expenditure in mice. . Diabetes 2009, 7.

13. Atabay VE, Lutfioğlu M, Avci B, Sakallioglu EE, Aydoğdu A: Obesity and oxidative stress in patients with different periodontal status: a case-control study. Journal of Periodontal Research 2017, 52:1221.

14. Bournat JC, Brown CW: Mitochondrial Dysfunction in Obesity. Current Opinion in Endocrinology Diabetes \& Obesity 2010, 17:446.

15. Petrill S, Pelosi L, Piemonte F, Travaglini L: Oxidative stress in Duchenne muscular dystrophy: focus on the NRF2 redox pathway. Oxford University Press 2017, 26:362-378.

16. Julien SG, Kim SY, Brunmeir R, Sinnakannu JR, Ge X, Li H, Ma W, Yaligar J, Kn BP, Velan SS, et al: Narciclasine attenuates diet-induced obesity by promoting oxidative metabolism in skeletal muscle. PLoS Biol 2017, 15:e1002597.

17. Zou D, Geng N, Chen Y, Ren L, Liu X, Wan J, Guo S, Wang S: Ranolazine improves oxidative stress and mitochondrial function in the atrium of acetylcholine-CaCl2 induced atrial fibrillation rats. Life Sciences 2016, 156:7-14.

18. Johri A, Chandra A, Beal MF: PGC-1a, mitochondrial dysfunction, and Huntington's disease. Free Radical Biology \& Medicine 2013, 62:37. 
19. Kang D, Sang HK, Hamasaki N: Mitochondrial transcription factor A (TFAM): Roles in maintenance of mtDNA and cellular functions. Mitochondrion 2007, 7:39-44.

20. Liu Y, Vertommen D, Rider MH, Lai YC: Mammalian target of rapamycin-independent S6K1 and 4EBP1 phosphorylation during contraction in rat skeletal muscle. Cellular Signalling 2013, 25:18771886.

21. Carneiro I, González T, López M, Señarís R, Devesa J, Arce VM: Myostatin expression is regulated by underfeeding and neonatal programming in rats. Journal of Physiology \& Biochemistry 2013, 69:1523.

22. Siu PM, Donley DA, Bryner RW, Alway SE: Myogenin and oxidative enzyme gene expression levels are elevated in rat soleus muscles after endurance training. Journal of Applied Physiology 2004, 97:277.

23. Gingras AA, White PJ, Chouinard PY, Julien P, Davis TA, Dombrowski L, Couture Y, Dubreuil P, Myre A, Bergeron K: Long-chain omega-3 fatty acids regulate bovine whole-body protein metabolism by promoting muscle insulin signalling to the Akt-mTOR-S6K1 pathway and insulin sensitivity. Journal of Physiology 2007, 579:269-284.

24. Schenk S, Mccurdy CE, Philp A, Chen MZ, Holliday MJ, Bandyopadhyay GK, Osborn O, Baar K, Olefsky JM: Sirt1 enhances skeletal muscle insulin sensitivity in mice during caloric restriction. Journal of Clinical Investigation 2011, 121:4281-4288.

25. Zhanguo G: Butyrate improves insulin sensitivity and increases energy expenditure in mice. Diabetes $2009,7$.

26. Peng L, Li ZR, Green RS, Holzman IR, Lin J: Butyrate Enhances the Intestinal Barrier by Facilitating Tight Junction Assembly via Activation of AMP-Activated Protein Kinase in Caco-2 Cell Monolayers. Journal of Nutrition 2009, 139:1619-1625.

27. Yan Z, Chen W, Liu S, Zhang G, Sun D, Hu S: Myocardial insulin signaling and glucose transport are up-regulated in Goto-Kakizaki type 2 diabetic rats after ileal transposition. Obesity Surgery 2012 , 22:493-501.

28. Li F, Cao H, Sheng C, Sun H, Song K, Qu S: Upregulated Pdx1 and MafA Contribute to beta-Cell Function Improvement by Sleeve Gastrectomy. Obes Surg 2016, 26:904-909.

29. Qi L, Si T, Xu X, Liang F, Wang L, Pan S: Electromagnetic radiation at $900 \mathrm{MHz}$ induces sperm apoptosis through bcl-2, bax and caspase-3 signaling pathways in rats. Reproductive Health 2015, 12:1-9.

30. An L, Wang Y, Wang C, Fan M, Han X, Xu G, Yuan G, Li H, Sheng Y, Wang M: Protective effect of Schisandrae chinensis oil on pancreatic $\beta$-cells in diabetic rats. Endocrine 2015, 48:818-825.

\section{Figures}


(a)

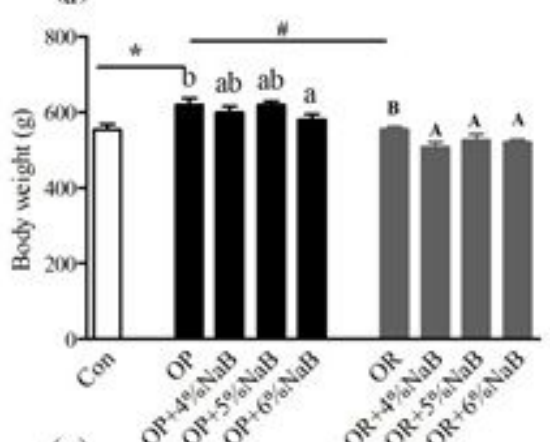

(c)

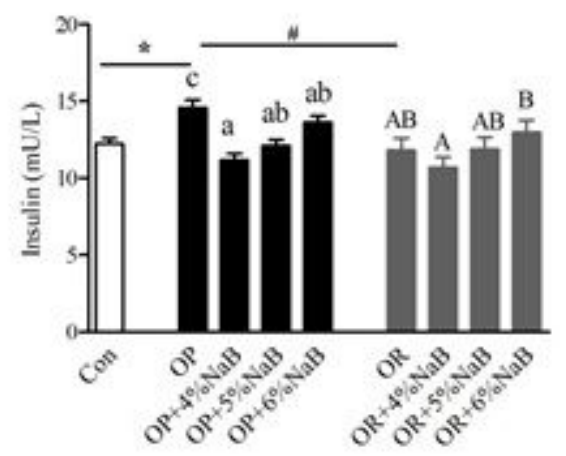

(b)

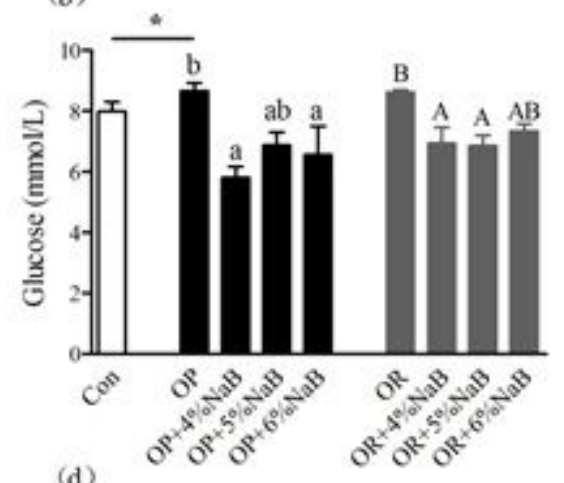

(d)

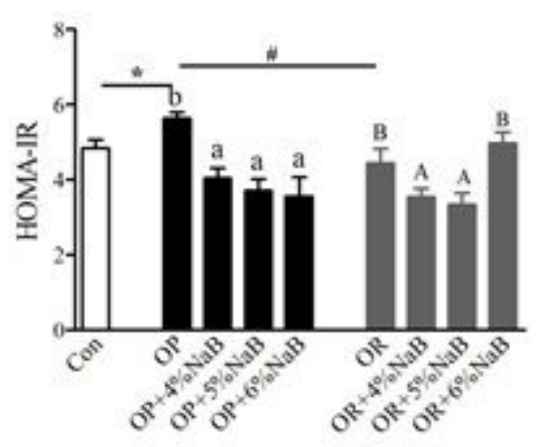

Figure 1

Effect of NaB on (a) body weight, (b) blood glucose, (c) insulin, and (d) insulin resistant index of high-fat diet (HFD) fed SD rats. Results are expressed as means \pm SE $(n=7)$. Con: control group; OP: obesity-prone group; $\mathrm{OP}+4 \% \mathrm{NaB}$ : obesity-prone supplemented with $4 \%$ sodium butyrate; $\mathrm{OP}+5 \% \mathrm{NaB}$ : obesity-prone supplemented with $5 \%$ sodium butyrate; $\mathrm{OP}+6 \% \mathrm{NaB}$ : obesity-prone supplemented with $6 \%$ sodium butyrate; OR: obesity-resistant group; OR+4\% NaB: obesity-resistant supplemented with $4 \%$ sodium butyrate; OR+5\% NaB: obesity-resistant supplemented with $5 \%$ sodium butyrate; OR+6\% NaB: obesityresistant supplemented with $6 \%$ sodium butyrate; * $\mathrm{P}<0.05$ compared with the control group, \# $\mathrm{P}<0.05$ compared with the OP group. Different letters indicate significant differences, $\mathrm{P}<0.05$. 

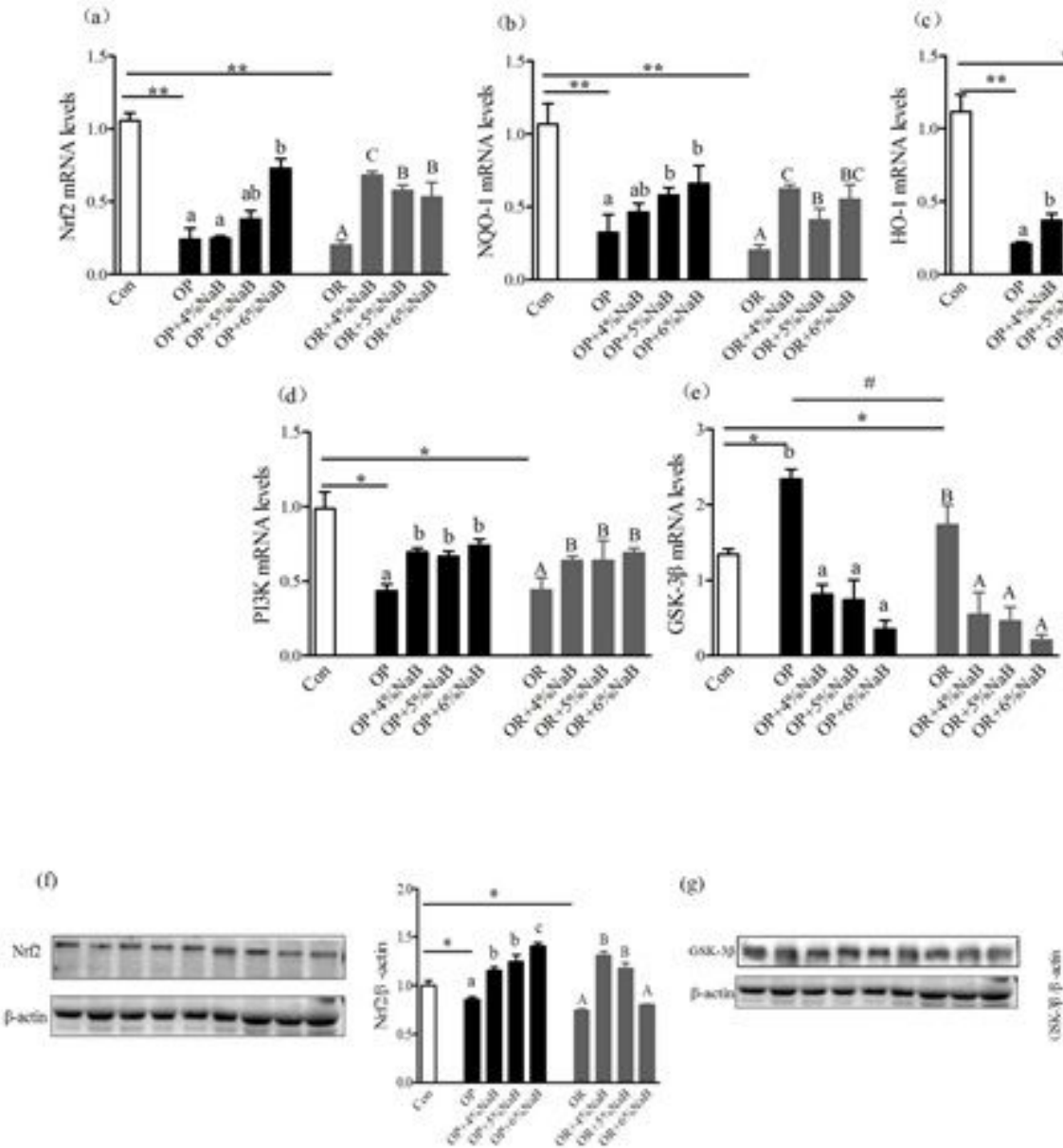

(g)
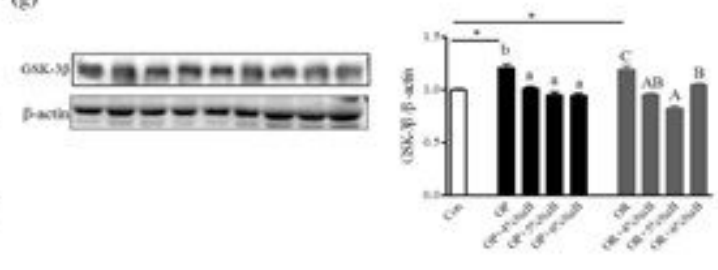

Figure 2

Effects of NaB on the expression of antioxidant genes (a) Nrf2, (b) NQO-1, (c) HO-1, (d) PI3K, (e) GSK3 and proteins expression (f) Nrf2 (g) GSK3 $\beta$ of gastrocnemius muscle in SD rats. Results are expressed as means $\pm S E(n=7)$. * $P<0.05$ versus the control group, \# $P<0.05$ versus the OP group. Different letters indicate significant differences, $P<0.05$. 

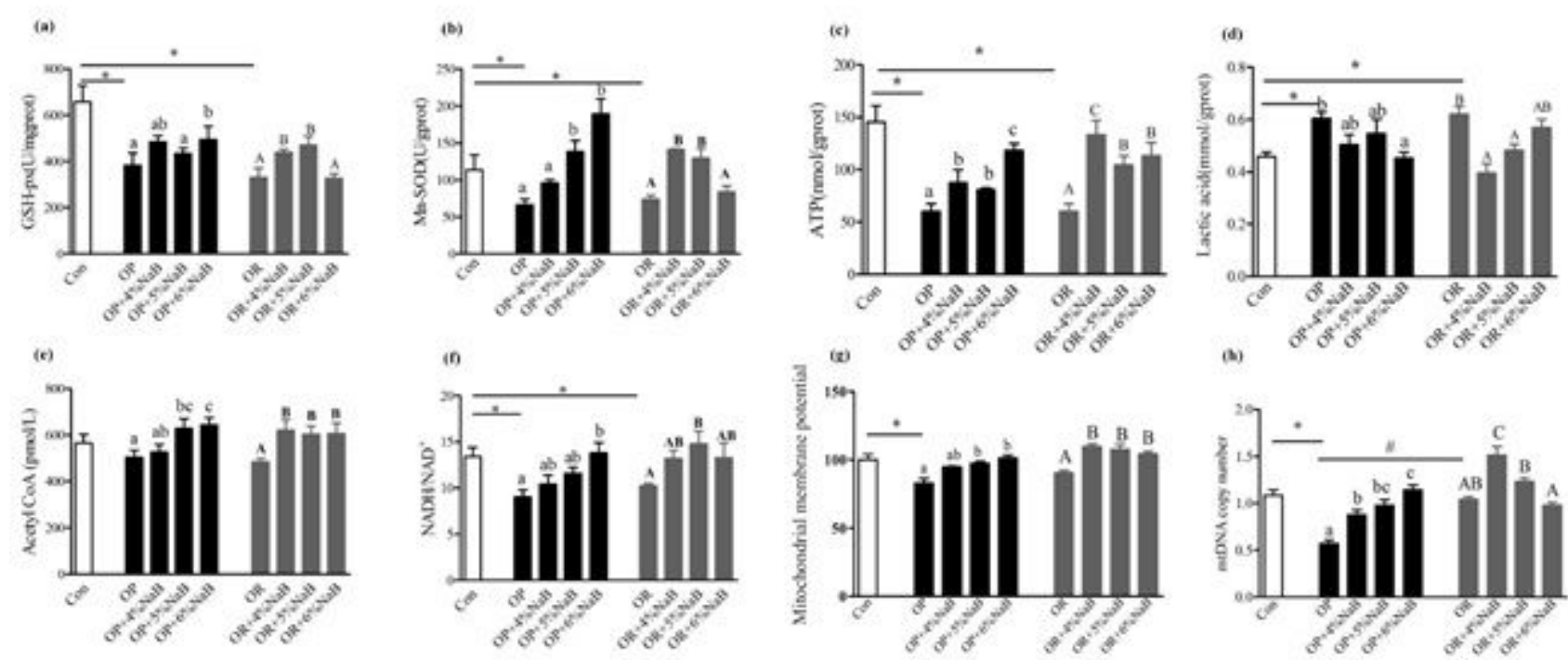

(a)
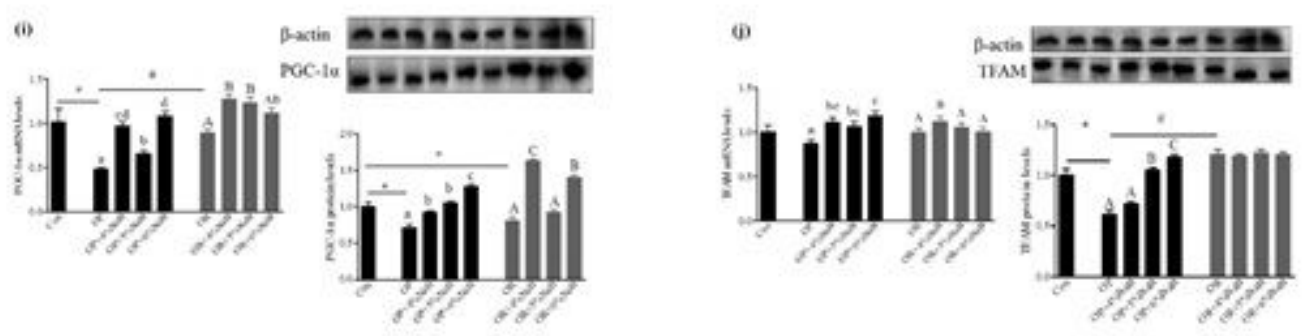

Figure 3

Effect of $\mathrm{NaB}$ on Mitochondrial function in gastrocnemius muscle in SD rats. (a) GSH-px, (b) Mn-SOD, (c) ATP, (d) Lactic acid, (e) Acetyl CoA, (f) NADH/NAD+, (g) Mitochondrial membrane potential, (h) mDNA copy number, (i) PGC-1 mRNA and protein expression, (j) TFAM mRNA and protein expression. Results are expressed as means $\pm S E(n=7) .{ }^{*} P<0.05$ versus the control group, $\# P<0.05$ versus the OP group. Different letters indicate significant differences, $\mathrm{P}<0.05$.
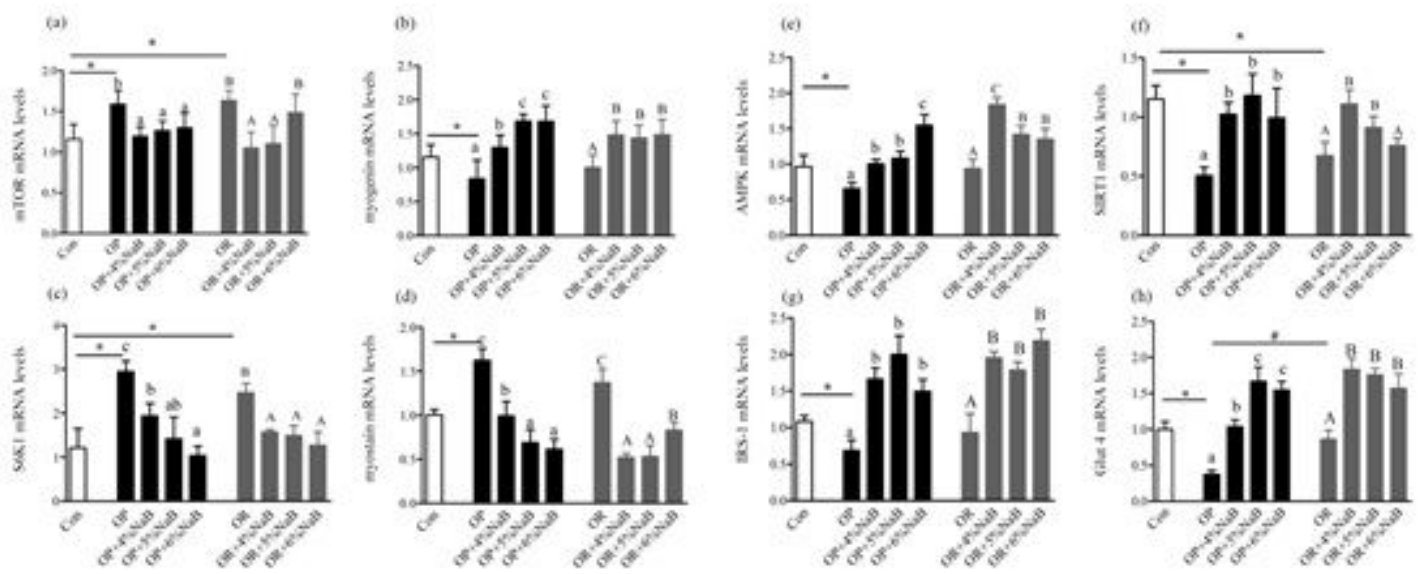

Figure 4 
Effect of $\mathrm{NaB}$ on muscle synthesis related gene expression. (a) mTOR, (b) myogenin, (c) S6K1, (d) myostain, (e) AMPK, (f) SIRT1, (g) IRS-1, (h) Glut4 mRNA expression. Results are expressed as means \pm $S E(n=7)$. $P<0.05$ versus the control group, $\# P<0.05$ versus the OP group. Different letters indicate significant differences, $\mathrm{P}<0.05$.

(a)

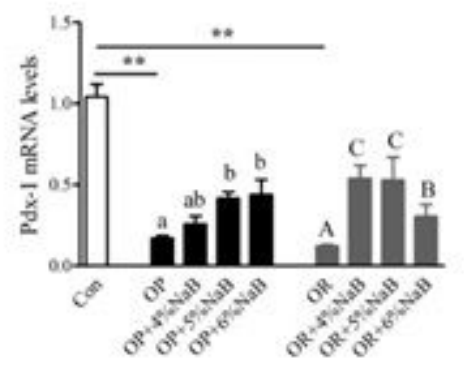

(b)

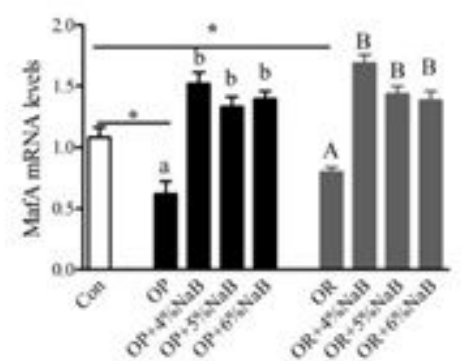

(c)

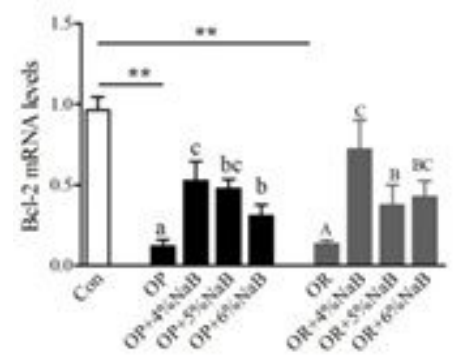

(d)

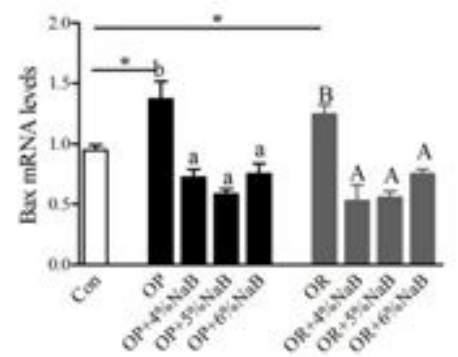

(c)

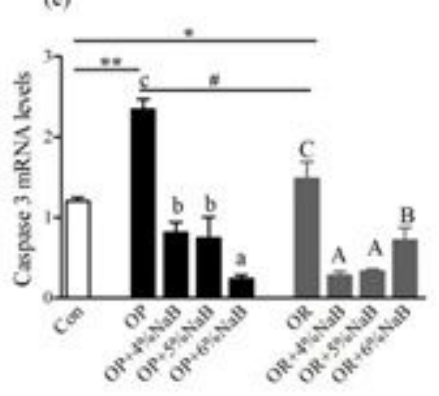

Figure 5

Effect of $\mathrm{NaB}$ on insulin-related gene expression in SD rats. (a) Pdx-1, (b) MafA, (c) Bcl-2, (d) Bax, (e) Caspase 3 mRNA expression. Results are expressed as means $\pm S E(n=7)$. ${ }^{*}<0.05$ versus the control group, \# $\mathrm{P}<0.05$ versus the OP group. Different letters indicate significant differences, $\mathrm{P}<0.05$. 